\title{
A neutral core of degressively proportional allocations under lexicographic preferences of agents
}

\author{
Katarzyna Cegiełka ${ }^{1}$ (D) Piotr Dniestrzański ${ }^{1}$ D Janusz Łyko $^{1}$ (D) \\ Arkadiusz Maciuk $^{1}$ - Maciej Szczeciński ${ }^{1}$ (D)
}

Received: 22 March 2020 / Revised: 15 March 2021 / Accepted: 5 April 2021 /

Published online: 22 July 2021

(c) The Author(s) 2021

\begin{abstract}
One of the main problems of practical applications of degressively proportional allocations of goods and burdens is lack of uniqueness of this principle. Even under given boundary conditions of allocation, i.e. determined minimal and maximal amounts of a good that can be assigned in a given allocation, there are usually many feasible solutions. The lack of formal rules of allocation is the reason why the allocation is typically a result of negotiations among its agents. A number of allocations favor some of agents or their groups, therefore other agents cannot accept them. The aim of this paper is to indicate a way of reducing the set of all feasible solutions exclusively to those that are neutral to all agents. As a result of the term of lexicographic preference of allocation agents defined on the basis of the relation theory followed by a numerical analysis of sets of all feasible solutions, it is possible to determine a core of this set in the form of a subset of all feasible solutions that are acceptable by all agents. In addition, this subset can be further divided into smaller subsets with regard to the degree of acceptance of their elements. Theoretical analysis is complemented by case studies, one of which is application of this idea to the allocation of seats in the European Parliament among the member states of the European Union.
\end{abstract}

Keywords Fair division - Lexicographic order · Degressive proportionality · European Parliament

JEL Classification D30 · D63 · D69

Katarzyna Cegiełka

katarzyna.cegielka@ue.wroc.pl

Extended author information available on the last page of the article 


\section{Introduction}

Allocation problems concern most aspects of the functioning of contemporary societies. Distribution methods must be decided by enterprises or communities as well as by government administrative units such as voivodeship, and also state and international organizations. In most cases, the allocation depends on the value of the agents involved in the division. These values, called entitlements, can be significantly diversified and in most cases are numerically defined. When dividing a company's profit for example, it is the number of shares held. On the other hand, in the election of social representation, it is the population of individual constituencies. In the case of shared costs of investment it is the degree of its use by investors. A decision-making problem arises when there is more than one allocation applicable to one allocation problem. As a result, the decision maker requires the assistance of some tools.

In the literature on the subject, there are many analysis trends regarding multicriteria decision support and decision aiding. One of them refers to the problem of numerical evaluation of acceptable alternatives from the perspective of given criteria and on this basis selecting solutions that can be accepted by the decisionmaker (Papathanasiou \& Ploskas, 2018; Xu, 2001; Yildirim \& Mercangoz, 2020). In this paper, a similar decision problem is considered in terms of order. Based on the order relation, the best and the worst alternatives for a given criterion are determined, and then by eliminating them, neutral elements that constitute an initial point for the next evaluation are found. Iterating this procedure does not always lead to a single solution, but in the case of a large set of alternatives, it significantly reduces its cardinality and thus facilitates negotiation or choice.

The conditions defining an allocation are typically derived from one of three general concepts of fairness. The first one is equal rights implying equal treatment of all agents. Next, proportionality involves the acceptance of differences between agents and the division of a good proportionately to those differences. The last concept is priority that demands to confer a good on the most entitled agent (Young, 1994). All these concepts determine the allocation without ambiguity. Assuming one of them leads directly to the solution of a division problem that precisely indicates the due amount of a divided good allocated to each agent. In the first case, each agent is given the same amount of the good. In the second one, the amount of allocated good is proportional to the ratio of value (claims, entitlements) of the given agent to the sum of all values of all agents participating in division. The third case allocates the whole resource to one agent that has got the highest priority over all contenders.

In some instances, however, the unambiguity does not hold. There are circumstances where the conditions defining an allocation to comply with a certain concept of fairness do not allow to unambiguously allocate respective amounts of goods to agents participating in division. A typical example is a degressively proportional division. According to its conditions the goods are distributed among agents depending on their values in such a way that the agents with greater values are given the number of goods greater than the agents with smaller values, but 
at the same time the number of allocated goods is limited so that the ratio of the number of goods received to the entitlements of an agent with greater claims should not exceed this ratio for an agent with smaller claims. Hence, when compared to the fairness concept called proportionality, a degressively proportional allocation favors the agents with smaller claims by giving them the right to a greater number of goods than they would receive in case of a proportional division. By contrast, from the viewpoint of the concept of equal rights, now agents with greater claims are more privileged.

The concept of degressive proportionality can be therefore located between classical concepts of equal rights and proportionality, and thus perceived as an indication of solidarity among all participants in division. They respect the differences resulting from their values and as a result, they relinquish equal rights, but at the same time, they choose proportionality, which may lead to the domination of agents with greatest values, and on the other hand, to marginalization of agents with smallest values. For this reason, one may interpret this case in such a way that agents with greater values transfer a part of their entitlements to those with smaller values for the sake of a more balanced division that is easier to be endorsed by the entire group and thereby achieving common goals can take precedence over particular interests of agents.

This concept was acknowledged by the leaders of the European Union and the Lisbon Treaty proclaimed that the allocation of seats in the European Parliament among member states has to be degressively proportional with respect to the number of population. Thanks to it, in spite of largely disproportional populations, a just representation was guaranteed to the smallest states in the community and their marginalization was avoided in decision taking on the most important issues concerning the European Union. The solution is consequently an indication of international solidarity and the good of all members of the community takes precedence over individual interests of member states.

However, the solution is flawed due to the aforementioned lack of unambiguity. Even in case of integer divisions subject to fixed boundary conditions, i.e. predetermined minimum and maximum number of goods available to respective agents, as is the task of allocation of seats in the European Parliament, the number of feasible solutions can be large. For example, when allocating the seats for the 2014-2019 term the cardinality of the set of all feasible solutions was more than five million (Arora et al., 2016; Łyko \& Rudek, 2013).

The lack of uniqueness implies that the required division depends on numeric quantities which characterize the participants in division and that is subject to predetermined conditions, therefore there are many feasible solutions. All participants in division are interested in being allocated as many goods as possible, hence various feasible divisions are differently valued by each of them. How to efficiently negotiate in this situation so as to determine one solution out of many possible outcomes? The problem is not just purely theoretical. In spite of imposing the principle of degressive proportionality by the Lisbon treaty in 2007, the European Parliament has not adopted so far any real, repeatable method to determine its composition, and each time the numbers of seats are decided in negotiations that is an inefficient task in view of many millions of potential solutions. 
The goal of this paper is to indicate a method which makes the decision process efficient by reduction of the cardinality of a set of divisions. Through ordering the set of all divisions and then precisely determining which of them favor and disfavor ordered sets (permutations), it is possible to indicate allocations which are neutral to all agents in a sense that they neither favor or disfavor anyone. The favoring and disfavoring allocations are understood as the maximal and minimal elements in a given relation, i.e. those that are most or least favorable for a given permutation of agents (see the sets Favour(X) and Disfavour(X) set in the Methodology section). By eliminating those allocations which favor or disfavor, the neutral ones can be found (see the set $\operatorname{Core}(\mathrm{X})$ in the Methodology section) and this limitation of negotiations to the set of neutral divisions significantly reduces the problem. A proposed iterative method of determining neutral divisions makes it possible to significantly reduce the cardinality of the set of all feasible solutions and consequently, to streamline the process of negotiations. The presented algorithm along with its interpretation of favoritism and disfavoritism can be used in any allocation problem. However, the motivation for its development was the problem of degressively proportional division of indivisible goods, therefore considerations mainly relate to this problem.

The main contribution of this paper is a reduction of the cardinality of a given set of degressively proportional allocations through the use of its properties as a set with lexicographical ordering. The authors, to the best of their knowledge, introduce the notion of lexicographical order with respect to permutation in the context of a degressively proportional allocation problem for the first time. Such an approach enables the reduction of the cardinality in such a way that extreme solutions are excluded and hence the remaining allocations are acceptable to every agent. Therefore, the decision-making process is improved. The main result of this paper is the identification of neutral allocations by defining the favoring and disfavoring ones and, as a consequence of eliminating them, obtaining a significant reduction of the cardinality of the set of feasible solutions while maintaining the status quo.

\section{Review of literature}

The literature on allocation problems is highly diversified. It applies, for example, to allocation of government spending across administrative units (Agenor \& Neanidis, 2011; Zang \& Zou, 1998), cost sharing problem (Chen et al., 2017; Pham, 2019; Rudek \& Heppner, 2020; Young, 1994) or problem of proportional representation (Leeman \& Mares, 2014; Pukelsheim, 2014; Young, 1994). One of the newer research directions of the allocation problems is the problem of degressively proportional allocations.

Researches into the issue of degressive proportionality became more intensified after its introduction to the Lisbon Treaty. The early papers were directly dealing with the division of seats in the European Parliament and mostly focused on proposing actual methods to determine degressively proportional allocations of seats in the European Parliament. Progressively the research became wider and degressive proportionality was analyzed from the viewpoint of its theoretical properties, apart from the European Parliament. Degressively proportional allocation is applied in 
many political, social and business institutions such as the US Electoral College, the Senate of Canada or the Governing Council of the European Central Bank, as well as in many university senates.

At present, the published papers can be classified into the three main streams. The first one deals with generally approached foundations of degressive proportionality. Its mathematical properties and potential applications in practice are studied. The idea of degressive proportionality, among others, is drawn from the Penrose square root law (Kurz et al., 2017; Miller, 2012). This approach is flawed by the requirement to assume that agent's preferences are independent and identically distributed. Another approach based on utility theory is presented in the paper by Koriyama et al. (2013),Barbera and Jackson (2006), Beisbart and Bovens (2007). It is generally assumed that utility functions of individual voters are increasing and concave, thus leading to a conclusion that the optimal apportionment is a degressively proportional division. In addition, the measures of degression of divisions or of families of degressively proportional divisions are introduced using, among others, measures of inequality and disproportionality. Furthermore, algorithms producing degressively proportional divisions are put forward. Among others, the papers (Arora et al., 2016; Charvat, 2019; Chen et al., 2017; Dniestrzański, 2014a, b; Dniestrzański \& Łyko, 2014; Haman, 2017; Łyko \& Rudek, 2013; Słomczyński \& Życzkowski, 2012) deal with those topics.

The next stream focuses on developing actual methods to determine degressively proportional divisions. Most papers published in this area can be organized in two main groups. The first one makes use of so-called allocation functions. Methods based on parabolic allocation functions, power allocation functions, piecewise linear allocation functions and other are constructed in this part (Dniestrzański, 2014a, 2014b; Grimmet et al., 2012; Haman, 2007; Martinez-Aroza \& Ramirez-Gonzalez, 2008; Pukelsheim, 2010; Ramirez-Gonzalez, 2012; Ramírez-González et al., 2006,2012 ). The second group is formed by proposals of divisions generated using numerical methods (Florek, 2012; Serafini, 2012).

The third stream of research encompasses papers dealing primarily with political aspects of degressive proportionality, i.e. the compliance of legal acts with the provisions of the Lisbon Treaty which introduced the degressive proportionality to the community (as in Łyko \& Łyko, 2020). A lot of research is also focused on one of the most important proposals developed in 2011 by the participants of the scientific meeting held at the instigation of the Committee on Constitutional Affairs of the European Parliament (AFCO) at the Centre for Mathematical Sciences, University of Cambridge. The papers such as Cegiełka et al. (2019), Duff (2012), Grimmett (2012), Grimmett et al. (2012), Habermas (2017), Haman (2017), Laslier (2012), Łyko and Rudek (2017), Macé and Treibich (2012), Moberg (2012), Pukelsheim and Grimmett (2018), Policy Department (2017) and Słomczyński and Życzkowski (2012) deal with this subject among other things.

Another approach to studying the properties of degressively proportional divisions can be found in Cegiełka et al. (2017). This paper presents the origin of a new research stream in the area of degressive proportionality. In it, a new concept of the so-called transfer order was introduced so as to order the set of all degressively proportional divisions, under some given boundary conditions, with respect to potential 
transfers of the units of a good being distributed among the division participants. The results contained in the paper have been further developed in Cegiełkaet al. ( 2019a), where the authors introduce an algorithm that finds the maximal element in the transfer order and hence indicates an allocation considered to be the nearest to proportional allocation. Both of the papers, by opening the ordering stream of a degressively proportional allocation problem, provide new perspective and tools for it. To the best of our current knowledge, the ordering properties of a set of all degressively proportional divisions have not been previously investigated. This paper also belongs to that stream. The newly proposed approach, based on analysis of lexicographical order with respect to any permutation, enables the indication of allocations which are neutral for all agents and makes it possible to look at the issue from the perspective of priorities and the order in which claims are satisfied, similar to procedures known from the literature on handling customers' claims problems. In this paper, unlike previous papers where the maximal elements of a fixed order were sought, all possible lexicographical orders are considered. Thus, a full study of all possible cases is done and allocations that neither favor nor disfavor any agent are found.

\section{Problem statement}

The apportionment problem arises whenever some amount of goods has to be divided among a group of allocation participants. A decision-making problem arises when there exist more than one feasible allocation and only one needs to be chosen. Such an issue is a problem of degressively proportional apportionment. For example, as already mentioned in the case of degressively proportional allocation of seats in the European Parliament, the cardinality of the set of all feasible allocations in the 2014-2019 term was more than five millions.

Formally, the apportionment problem can be specified as follows. Let $n$ be a number of participants of the allocation (agents), $v=\left(v_{1}, v_{2}, \ldots, v_{n}\right), v_{i}>0$-sequence of real values characterizing agents and $H>0$-amount of goods to be divided. The problem is to allocate $H$ goods among $n$ agents, i.e. based on the sequence $v=\left(v_{1}, v_{2}, \ldots, v_{n}\right)$, to find a sequence $a=\left(a_{1}, a_{2}, \ldots, a_{n}\right)$ of nonnegative reals such that $\sum_{i=1}^{n n} a_{i}=H$. If a sequence $a=\left(a_{1}, a_{2}, \ldots, a_{n}\right)$ is nonnegative and integer, then the allocation is called integer.

In many cases the underlying conditions make it possible to find the sequence $a=\left(a_{1}, a_{2}, \ldots, a_{n}\right)$ in a unique way. We deal then with a so-called allocation rule, i.e. any function $R: \mathbb{R}_{+} \times \mathbb{R}_{+}^{n} \rightarrow A, \quad$ where $A=\left\{\left(a_{1}, a_{2}, \ldots, a_{n}\right) \in \mathbb{R}_{\geq 0}^{n}: \sum_{i=1}^{n} a_{i}=H\right\}$ (Pukelsheim, 2014). The allocation rules, as functions, always return a unique solution to the problem. For every pair $(H, v)$ there exists exactly one sequence $a=\left(a_{1}, a_{2}, \ldots, a_{n}\right)$ that is a realization of this rule. For example, in case of classical concepts of fairness we have for equal 
rights, proportionality and priority, respectively: $R_{u}(H, v)=\left(\frac{H}{n}, \frac{H}{n}, \ldots, \frac{H}{n}\right)$, $R_{p}(H, v)=\left(v_{1} \frac{H}{\sum_{j=1}^{n} v_{j}}, v_{2} \frac{H}{\sum_{j=1}^{n} v_{j}}, \ldots, v_{n} \frac{H}{\sum_{j=1}^{n} v_{j}}\right), R_{p r}(H, v)=(0, \ldots, 0, H, 0, \ldots, 0)$.

However, it may happen that the conditions of allocation do not allow to find a unique sequence $a=\left(a_{1}, a_{2}, \ldots, a_{n}\right)$, and instead they lead to a so-called set of feasible solutions. Then one deals with an allocation criterion that can be formally defined as a function whose values are subsets of the set $\left\{\left(a_{1}, a_{2}, \ldots, a_{n}\right) \in \mathbb{R}_{\geq 0}^{n}: \sum_{i=1}^{n} a_{i}=H\right\}$, i.e. the function $K: \mathbb{R}_{+} \times \mathbb{R}_{+}^{n} \rightarrow P(A)$. The value of such a function is above-mentioned set of feasible solutions. This set can be empty and then there is no allocation satisfying given conditions. When the set of feasible solutions is not empty, the allocation satisfying predetermined conditions is possible, but except for the case of a set with exactly one element, it cannot be determined uniquely. What are the possibilities to practically perform a division in such a case? One approach is to specify the allocation conditions more precisely, so that the value of the allocation criterion could always be a set with one element, i.e. in reality the allocation criterion becomes the allocation rule. However, the allocation conditions must be changed and the underlying concept of fairness could be violated. In some cases therefore it is not viable. Another possibility is to let the interested participants in division select one element from the set of feasible solutions. This option leads to negotiations among agents to ensure each of them the position as good as possible, i.e. mostly the allocation of the largest number of goods.

Formally approached, the general conditions of degressively proportional allocation are defined by two sequences of inequalities:

1. if $v_{1} \leq v_{2} \leq \cdots \leq v_{n}$ then $a_{1} \leq a_{2} \leq \cdots \leq a_{n}$ (monotonicity),

2. if $v_{1} \leq v_{2} \leq \cdots \leq v_{n}$ then $\frac{a_{1}}{v_{1}} \leq \frac{a_{2}}{v_{2}} \leq \cdots \leq \frac{a_{n}}{v_{n}}$ (degression),

with additional assumptions for the case of division of seats in the European Parliament, i.e. the solution must be integer, and the boundary conditions for $m$ and $M$, defining the minimal and maximal number of seats available to member states. As a result, the allocation criterion determines the set of feasible solutions $D P$. The cardinality of this set obviously depends on parameters $H, v, m$, and $M$, whose values for the division of seats in the European Parliament of its past three terms used to vary between several millions to several hundreds of millions.

As of today, there have been no decision yet regarding a more precise definition of allocation conditions to generate the set of feasible solutions with exactly one element, therefore all previous allocations of seats were results of political negotiations. One is not able to imagine that they can be precise and effective, when there are many millions of feasible solutions at hand. For this reason, our paper puts forward an algorithm to significantly reduce the cardinality of the set of feasible solutions without detriment to the choice of optimal allocation, accepted by all agents. Such reduction is achieved by eliminating those allocations which cannot be accepted by agents because they either favor or disfavor 
some agents. First, the algorithm will be presented generally and then it will be applied, inter alia, to the task of allocating seats in the European Parliament for its first term.

\section{Methodology}

The fundamental idea employed in the remainder in order to reduce the cardinality of the set of all feasible solutions is to distinguish allocations which favor and disfavor particular agents. Favoring is meant as allocation of the maximal number of distributed goods available under given conditions. By contrast, disfavoring means that the minimal available number of goods is allocated. Certainly, more than one agent can be allocated either maximal or minimal number of goods and that is why the problem is considered in the context of lexicographically ordered set of all agents. Lexicographical ordering of agents specifies here the succession in which their claims are satisfied. This succession is not relevant for the final solution but significantly simplifies the formal description of the problem.

A lexicographical order in set $\mathbb{R}^{n}$ is called the relation $\leq_{L}$ defined as follows:

$$
\begin{gathered}
x \leq_{L} y \Leftrightarrow\left(\left(x_{1}<y_{1}\right) \vee\left(x_{1}=y_{1} \wedge x_{2}<y_{2}\right) \vee \cdots \vee\right. \\
\left.\left(x_{1}=y_{1} \wedge x_{2}=y_{2} \wedge \cdots \wedge x_{n-1}=y_{n-1} \wedge x_{n} \leq y_{n}\right)\right) .
\end{gathered}
$$

Let $[n]=\{1,2, \ldots n\}$ be a set of $n$ first natural numbers. Symbol $S_{n}$ will denote the set of all permutations of the set $[n]$, i.e. $S_{n}=\{\theta:[n] \rightarrow[n]: \theta$ is bijective $\}$. For $\theta \in S_{n}$ and $x=\left(x_{1}, x_{2}, \ldots x_{n}\right) \in \mathbb{R}^{n}$ we define $x^{\theta}=\left(x_{\theta(1)}, x_{\theta(2)}, \ldots, x_{\theta(n)}\right)$. A lexicographical order with respect to permutation $\theta$ will be called the relation $\leq_{\theta L}$ defined as follows:

$$
\begin{aligned}
& x \leq_{\theta L} y \Leftrightarrow\left(\left(x_{\theta(1)}<y_{\theta(1)}\right) \vee\left(x_{\theta(1)}=y_{\theta(1)} \wedge x_{\theta(2)}<y_{\theta(2)}\right) \vee \cdots \vee\right. \\
& \left.\left(x_{\theta(1)}=y_{\theta(1)} \wedge x_{\theta(2)}=y_{\theta(2)} \wedge \cdots \wedge x_{\theta(n-1)}=y_{\theta(n-1)} \wedge x_{\theta(n)} \leq y_{\theta(n)}\right)\right) .
\end{aligned}
$$

In case when relation $x \leq_{\theta L} y$ holds, $x$ is said to be smaller than $y$ with respect to relation $\leq_{\theta L}$ or $y$ is said to be greater than $x$ with respect to relation $\leq_{\theta L}$.

An element from the non-empty and finite set $X \subset \mathbb{R}^{n}$, which is greater than all elements of the set $X$ with respect to relation $\leq_{\theta L}$ is said to be a maximal element in the set $X$ with respect to relation $\leq_{\theta L}$ and is denoted by $\max \left(X, \leq_{\theta L}\right)$. Similarly we define a minimal element in the set $X$ with respect to relation $\leq_{\theta L}$ which will be denoted by $\min \left(X, \leq_{\theta L}\right)$. Obviously, since the considered set $X$ is non-empty and finite and a lexicographical order is a linear order, there always exists exactly one maximal element in every $\left(X, \leq_{\theta L}\right)$. The same is true for minimal elements. It should be also intuitively clear that, aside from trivial cases, there does not exist an element in $X$ which is a maximal (minimal) element in every permutation (as in examples in Sect. 5.1). However, there may exist an element in $X$ which is a maximal (minimal) element for more than one permutation (as $a_{1}$ in first example in Sect. 5.1).

A set of all maximal elements with respect to some permutation will be denoted by $\operatorname{Favor}(X)$ : 


$$
\operatorname{Favor}(X)=\bigcup_{\theta \in S_{n}}\left\{\max \left(X, \leq_{\theta L}\right)\right\} .
$$

The set Favor $(X)$ contains all elements that are maximal with respect to some relation $\leq_{\theta L}$. Since for every permutation there is exactly one maximal element, the set $\operatorname{Favor}(X)$ is non-empty for every non-empty set $X$. If the cardinality of $X$ is equal to $n, n \in \mathbb{N}$, then the set Favor $(X)$ contains at most $n$ ! elements, since there are $n$ ! permutations of a set of cardinality $n$ and, as mentioned earlier, there may exist an element which is maximal with respect to more than one lexicographical order relation. This is the case in Sect. 5.1. Analogous considerations apply to the following set Disfavor $(X)$.

A set of all minimal elements with respect to some permutation will be denoted by $\operatorname{Disfavor}(X)$ :

$$
\operatorname{Disfavor}(X)=\bigcup_{\theta \in S_{n}}\left\{\min \left(X, \leq_{\theta L}\right)\right\} .
$$

Let Neutral : $X \rightarrow P(X)$ be a function assigning to the set $X$ the set

$$
\operatorname{Neutral}(X)=X \backslash(\operatorname{Favor}(X) \cup \operatorname{Disfavor}(X)) \text {. }
$$

Let us assume by definition that $C_{0}(X)=X$. The set $\operatorname{Neutral}(X)$ includes the elements from the set $X$, which are neither maximal nor minimal with respect to any permutation. The set $\operatorname{Neutral}(X)$ is said to be the first core of the set $X$ and is denoted by $C_{1}(x)$. Hence, $C_{1}(X)=\operatorname{Neutral}(X)$. The set $C_{k}(X)=\operatorname{Neutral}\left(C_{k-1}(X)\right)$ is said to be the $k$ th core of the set $X$. It follows from construction of the sets $C_{k}(X)$ that for every set $X \subset \mathbb{R}^{n}$ there exists a natural number $k$, such that the set $C_{k}(X)$ is empty. If $\bar{k}$ is the largest natural number for which the set $C_{\bar{k}}(X)$ is nonempty, then the set $C_{\bar{k}}(X)$ is said to be a core of the set $X$ and is denoted by $\operatorname{Core}(X)$.

Definition of set $\operatorname{Core}(X)$ is recursive. It contains those elements of set $X$ that are neither maximal nor minimal with respect to any relation $\leq_{\theta L}$ at any step of the recursion. Elements of set $\operatorname{Core}(X)$ may therefore be considered as the most neutral, i.e. which least favor any permutation $\theta$.

\section{Results}

It can be easily noticed that if $X$ is the set of all allocations meeting some allocation criterion, then $\operatorname{Core}(X)$ can be interpreted as a set of divisions which are as neutral as possible for all participants of division. Indeed, because at each step of recursively working algorithm divisions $a^{\theta}=\left(a_{\theta(1)}, a_{\theta(2)}, \ldots, a_{\theta(n)}\right)$ which either favor or disfavor any sequence of agents for permutation $\theta \in S_{n}$ are eliminated. Favoring or disfavoring mean that a given division $a^{\theta}$ is a maximal or a minimal element, respectively, in the set $C_{k}(X)$ with respect to relation $\leq_{\theta L}$. At the final step of algorithm the core of the set $X$ is determined, i.e. the set $\operatorname{Core}(X)=C_{\bar{k}}(X)$. Assuming that during negotiations aimed at the selection of one division out of many feasible solutions no agent will either accept the allocation of the minimal number of goods available or 
veto the allocation of the maximal amount to another agent, then the core of the set is a natural subset of solutions to be considered by negotiators.

\subsection{Examples}

Let us consider a task of division of 14 goods among four agents, whose values are represented by a nondecreasing sequence $v=\left(v_{1}, v_{2}, v_{3}, v_{4}\right)$. The allocation criterion determining the set of all feasible solutions $X$ includes the condition of monotonicity as well as the constraints on minimum and maximum of goods available to agents. The constraints are defined by sequences $\min (X)=(1,1,1,4)$ and $\max (X)=(3,4,6,11)$. For example, agent one can be allocated between 1 and 3 goods, similarly other agents.

Table 1 illustrates the effect of algorithm working in the set $X$. Division one $a_{1}=(3,3,4,4)$ is the maximal division for relation defined by permutation 1324 and permutation 1342 (abbreviated as $13+$; symbol + denotes that it is the maximal element, whereas the lack of numbers of remaining two agents implies that it is maximal for any permutation of them) and permutation 1234. Division $a_{1}$ belongs therefore to the set of favoring divisions. It is also the minimal division for relations defined by permutation 4213 and permutation 4231 (analogously denoted as 42-) and by permutation 4321. Hence, it is also a disfavoring division. At the first step of the algorithm this division is eliminated, likewise divisions two, three, ten, eleven, eighteen and twenty-three. As a result, the initial set is reduced by elimination of seven divisions, therefore $C_{1}(X)$ will contain sixteen divisions. At step two of algorithm ten divisions are eliminated, thus $C_{2}(X)$ contains just six divisions. After applying such reduction for the third time, all remaining divisions will be eliminated. The core of this set is therefore the set $C_{2}(X)$ containing divisions $7,815,16,20$ and 21 . Hence, the negotiations about the selection of one division out of twenty-three feasible solutions can be limited to just six elements.

One can prove that the presented algorithm, under some additional assumptions on the set of divisions $X$, leads to a very intuitive solution known as the Maimonides' rule for rare goods. Indeed, if the number $H$ equals the sum of constraints regarding the minimal number of goods and a certain natural constant $b$, then $\operatorname{Core}(X)=\min (X)+b$. In other words, if there are $H$ indivisible goods to be divided among $n$ agents, then if there exists a division allocating to each agent the minimal number of goods available, and the surplus is divided equally among all agents, then this division is the single element of the set $\operatorname{Core}(X)$.

Table 2 presents the algorithm working to find the core of the set $X$, where $\min (X)=(1,3,5,7), \max (X)=(3,5,7,9) \quad$ and $H=20=(1+1)+(3+1)+(5+1)+(7+1)$. It can be easily seen that in this case $\operatorname{Core}(X)=\{(2,4,6,8)\}=\{\min (X)+1\}$, and it is both the mean and the median of coordinates of the set $X$. 
Table 1 Algorithm working example

\begin{tabular}{|c|c|c|c|c|c|c|c|}
\hline & 1 & 2 & 3 & 4 & 1 & 2 & 3 \\
\hline$a_{1}$ & 3 & 3 & 4 & 4 & $13+, 123+, 42-, 432-$ & & \\
\hline$a_{2}$ & 3 & 3 & 3 & 5 & $14+, 124+$ & & \\
\hline$a_{3}$ & 2 & 4 & 4 & 4 & $21+, 231+, 41-, 431-$ & & \\
\hline$a_{4}$ & 2 & 3 & 4 & 5 & & $\begin{array}{l}123+, 213+ \\
231+, 43-\end{array}$ & \\
\hline$a_{5}$ & 2 & 3 & 3 & 6 & & $124+, 214+$ & \\
\hline$a_{6}$ & 2 & 2 & 5 & 5 & & $\begin{array}{c}13+, 31+, 321+ \\
42-, 41-\end{array}$ & \\
\hline$a_{7}$ & 2 & 2 & 4 & 6 & & & Core \\
\hline$a_{8}$ & 2 & 2 & 3 & 7 & & & Core \\
\hline$a_{9}$ & 2 & 2 & 2 & 8 & & $14+, 34-$ & \\
\hline$a_{10}$ & 1 & 4 & 4 & 5 & $24+, 234+$ & & \\
\hline$a_{11}$ & 1 & 3 & 5 & 5 & $142-$ & & \\
\hline$a_{12}$ & 1 & 3 & 4 & 6 & & $234+, 143-$ & \\
\hline$a_{13}$ & 1 & 3 & 3 & 7 & & $24+$ & \\
\hline$a_{14}$ & 1 & 2 & 5 & 6 & & $34+, 324+, 142-$ & \\
\hline$a_{15}$ & 1 & 2 & 4 & 7 & & & Core \\
\hline$a_{16}$ & 1 & 2 & 3 & 8 & & & Core \\
\hline$a_{17}$ & 1 & 2 & 2 & 9 & & $\begin{array}{c}412+, 42+, 134-, \\
312-\end{array}$ & \\
\hline$a_{18}$ & 1 & 1 & 6 & 6 & $3+, 124-, 214-, 24-$ & & \\
\hline$a_{19}$ & 1 & 1 & 5 & 7 & & $\begin{array}{c}34+, \text { 124-, } 214-, \\
24-\end{array}$ & \\
\hline$a_{20}$ & 1 & 1 & 4 & 8 & & & Core \\
\hline$a_{21}$ & 1 & 1 & 3 & 9 & & & Core \\
\hline$a_{22}$ & 1 & 1 & 2 & 10 & & $\begin{array}{c}4+, 123-, 132- \\
23-, 213-\end{array}$ & \\
\hline$a_{23}$ & 1 & 1 & 1 & 11 & $4+, 123-, 213-, 23-, 3-$ & & \\
\hline
\end{tabular}

\section{Case studies}

In this section, we describe examples of practical cases that can be supported by the proposed method. Since the motivation for its development was the problem of degressively proportional division of indivisible goods we begin this section with its source, i.e. the problem of allocation of seats in the European Parliament. Next, as the algorithm can be used in any allocation problem, we present two additional cases-allocation of company shares and allocation of slots for FIFA World Cup. 


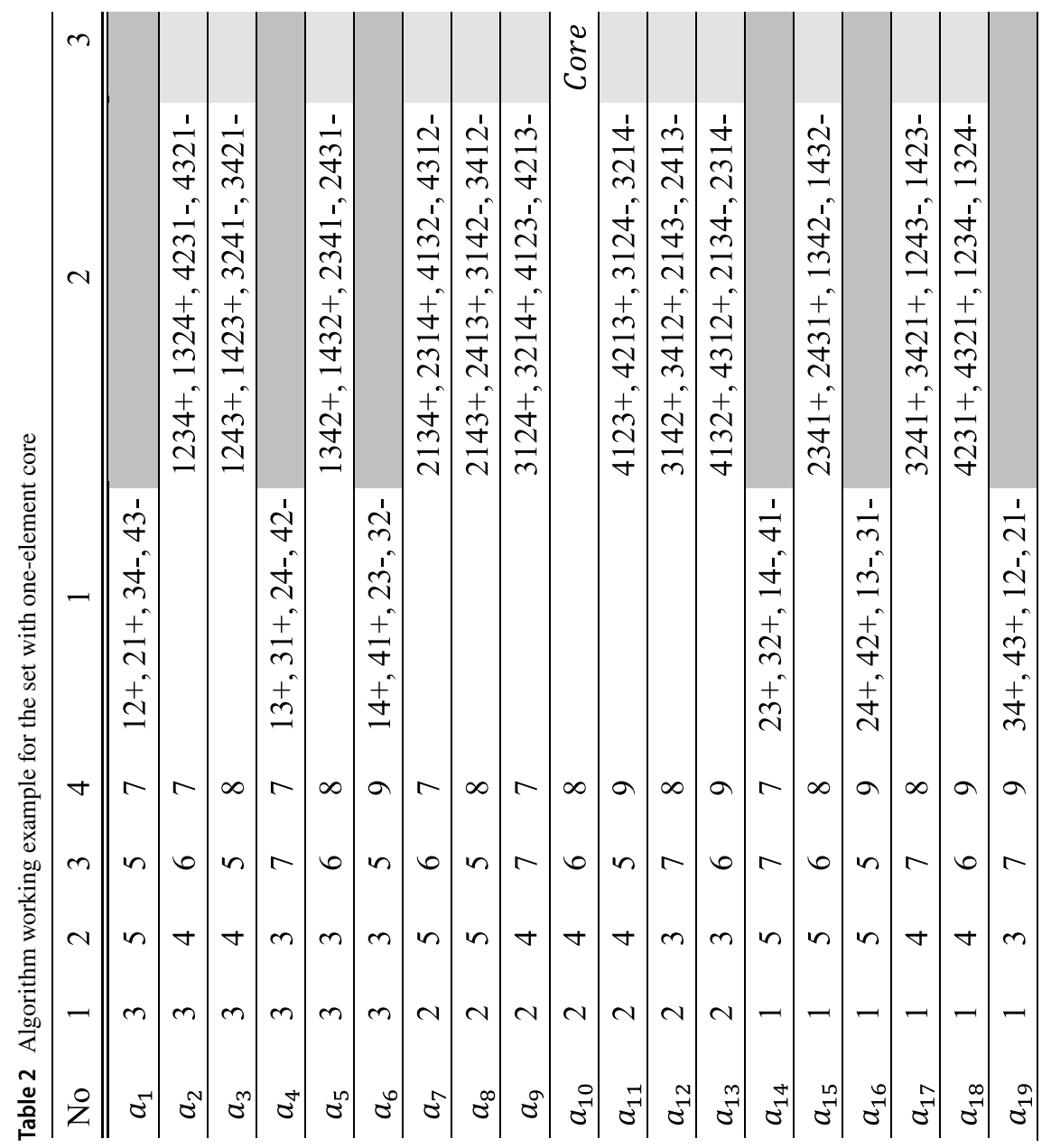




\subsection{Allocation of seats in the European Parliament}

At first, we applied the algorithm presented in this paper to determine the core of the set $D P$ of all feasible allocations of the seats in the European Parliament for its first term beginning in 1979. According to legal rules there were 410 seats to be allocated among nine countries, whereas a less populated country could not obtain more seats than a more populated country. There were also requirements that no country could obtain less than six seats and that more populated countries get less seats compared to the proportionality principle, hence the adopted division satisfied the conditions of proportional allocation. One can also assume, although it was not explicitly written in any document, that the boundary conditions $m=6$ and $M=81$ seats were followed.

The numbers of populations in member countries were given by sequence $v=(360.9 ; 3,228 ; 5,065.3 ; 9,811 ; 13,770 ; 54,159 ; 55,839 ; 52,216.1 ; 61,644.6)$

Table 3 presents the binding division of seats (row 2). Further rows of this table show the results of the algorithm proposed in this paper. First, the set was determined of all degressively proportional divisions of 410 seats among nine countries with values corresponding to numbers of their populations.

As a result, the set of feasible solutions with 7177 elements was obtained. Rows three and four of Table 3 present the values of sequences $\min (D P)$ and $\max (D P)$. A procedure being a part of the algorithm was performed 42 times. Consequently, the core $\operatorname{Core}(D P)=C_{42}(D P)$ with 6 elements was produced (rows 4-8 in Table 3).

In all allocations from the set $\operatorname{Core}(D P)$ four countries: Germany, the UK, Italy and Luxemburg are allocated the same numbers of seats (Germany-81, the UK and Italy - 77, Luxemburg-6). In case of Luxemburg and Germany the numbers results from the boundary conditions, therefore the allocations from the core are both favoring and disfavoring for two remaining countries, i.e. those countries can be neutral in negotiations.

Based on the values of sequences of minimal and maximal elements (see Table 3, rows 3 and 4) one can notice that the differences for the remaining countries as regards the numbers of allocated seats are one (Ireland, Denmark, Belgium) or two (Netherlands). In addition, for two countries (Ireland and Netherlands) the greatest

Table 3 Core $(D P)$ for the European Parliament as of 1979

\begin{tabular}{llllllllll}
\hline & Lux & Irl & Den & Bel & Ned & Fra & Ita & UK & Ger \\
\hline$a$ & 6 & 15 & 16 & 24 & 25 & 81 & 81 & 81 & 81 \\
$\min$ & 6 & 9 & 13 & 19 & 21 & 72 & 74 & 74 & 81 \\
$\max$ & 6 & 25 & 28 & 35 & 43 & 81 & 81 & 81 & 81 \\
$a_{1}$ & 6 & 16 & 19 & 26 & 32 & 76 & 77 & 77 & 81 \\
$a_{2}$ & 6 & 16 & 19 & 27 & 31 & 76 & 77 & 77 & 81 \\
$a_{3}$ & 6 & 16 & 20 & 27 & 30 & 76 & 77 & 77 & 81 \\
$a_{4}$ & 6 & 16 & 20 & 26 & 31 & 76 & 77 & 77 & 81 \\
$a_{5}$ & 6 & 17 & 19 & 26 & 31 & 76 & 77 & 77 & 81 \\
$a_{6}$ & 6 & 16 & 20 & 27 & 31 & 75 & 77 & 77 & 81 \\
\hline
\end{tabular}


number of allocated seats emerges only once, respectively in $a_{5}$ for Ireland and in $a_{1}$ for Netherlands. For this reason, the allocation $a_{5}$ is most advantageous for Ireland, and $a_{1}$ for Netherlands. Likewise, the division $a_{3}$ is the only allocation that is least advantageous for Netherlands, and $a_{6}$ is least advantageous for France (it is worth noting that both these divisions allocate the greatest number of seats to Denmark).

The binding division of seats in the European Parliament was the allocation $a=(6,15,16,24,25,81,81,81,81)$. This division is not an element of the set Core $(D P)$. It turns out that $a \in C_{11}(D P)$, hence it is far away from the core of the set $D P$. Furthermore, when comparing the actual allocation of seats in the European Parliament in 1979 with the set $\operatorname{Core}(D P)$ it is obvious that Ireland, Denmark, Belgium and Netherlands were allocated less seats than they could get if any division from the set $\operatorname{Core}(D P)$ would have been selected. France, Italy and the UK in contrast were allocated more seats than any division from this set would allocate. One can therefore assert that the division $a$ favors three largest countries (excluding Germany) and at the same time it disfavors four smallest countries (excluding Luxemburg). However, notice that not every allocation giving more seats to smaller countries needs to be in the set $\operatorname{Core}(D P)$. Such an allocation may, for example, favor middle sized countries and hence be eliminated at some step of the algorithm procedure (compare for example allocation $a_{1}$ in the second example in Sect. 5.1). Therefore, not every allocation that gives more seats to small countries than the actual division is neutral in the considered sense.

\subsection{Issue of company shares}

The second example of a practical application of the method proposed in this paper is the allocation of company shares to employees. Among many different problems of this type, let us consider the following example. Company $\mathrm{X}$ plans to issue shares. A part of the shares, treated as an element of motivation, is to be allocated to employees, where 60 of the shares are intended for employees of the sales department. The head of the department is to divide the shares among 5 employees with respect to the length of their employment in such a way that each employee is guaranteed 4 shares and none of the employees receives more than 20 shares. Additionally, to motivate employees with shorter lengths of employment to greater involvement in the company's results, the amount of shares per each year of employment cannot increase with an increase in the length of employment. Suppose further that lengths of employment are given by the sequence $v=(2,3,7,10,15)$. Clearly, the described example is a problem of degressively proportional allocation of $H=60$ goods with boundary conditions $m=4$ and $M=20$.

Table 4 presents the results of the algorithm proposed in the paper applied to this example. First, the set of 222 feasible solutions with respect to sequence $v$ together with the values of sequences $\min (D P)$ and $\max (D P)$ (rows one and two, respectively) were determined. Then, the procedure determining the core was performed 6 times. As a result, $\operatorname{Core}(D P)=C_{6}(D P)$ with 12 elements was produced (rows 3-15 in Table 4). 
Table $4 \operatorname{Core}(D P)$ for the example of shares issue

\begin{tabular}{llllll}
\hline & Employee 1 & Employee 2 & Employee3 & Employee 4 & Employee 5 \\
\hline $\min$ & 4 & 6 & 10 & 11 & 12 \\
$\max$ & 12 & 12 & 16 & 18 & 20 \\
$a_{1}$ & 7 & 8 & 13 & 14 & 18 \\
$a_{2}$ & 7 & 8 & 13 & 15 & 17 \\
$a_{3}$ & 7 & 9 & 12 & 14 & 18 \\
$a_{4}$ & 7 & 9 & 12 & 15 & 17 \\
$a_{5}$ & 7 & 9 & 13 & 14 & 17 \\
$a_{6}$ & 7 & 9 & 13 & 15 & 16 \\
$a_{7}$ & 7 & 10 & 12 & 14 & 17 \\
$a_{8}$ & 8 & 9 & 12 & 14 & 17 \\
$a_{9}$ & 8 & 9 & 12 & 15 & 16 \\
$a_{10}$ & 8 & 9 & 13 & 14 & 16 \\
$a_{11}$ & 8 & 10 & 12 & 13 & 17 \\
$a_{12}$ & 8 & 10 & 12 & 14 & 16 \\
\hline
\end{tabular}

\subsection{Allocation of slots for FIFA World Cup}

Another example is the allocation of slots for the FIFA World Cup (WC). From 2026, 48 countries will participate in the WC. According to FIFA's decision, two slots were reserved for the winners of a special intercontinental qualifying tournament, and the remaining 46 slots were distributed among six confederations representing different regions of the world: UEFA (Europe), CAF (Africa), CONCACAF (North and Central America), CONMEBOL (South America), OFC (New Zealand and South Pacific island nations) and AFC (Asia). Since one of the most important parameters describing the representational strength of FIFA's members is the number of points (and the related position) in the FIFA World Ranking, it was assumed that it would also be the basis for the analysis. The sum of the ranking points of all its members on May 4, 2017 (this ranking was valid in the month of the FIFA decision concerning the allocation of WC slots between confederations) was taken as the value characterizing given confederation. Moreover, the boundary conditions $m=1$ and $M=16$ were adopted, i.e. each confederation must receive at least one slot and no one may receive more than 16 slots. The upper boundary is consistent with the greatest number of slots allocated by FIFA, which prevents the dominance of one of the confederations. Thus, a set of 43 feasible solutions was obtained. The third and fourth rows of Table 5 present the values of the $\min (D P)$ and $\max (D P)$ sequences. The procedure being a part of the algorithm was performed 5 times. As a result, the $\operatorname{Core}(D P)=C_{5}(D P)$ with only one element was obtained (row 5 of Table 5).

The allocation established by FIFA $(a=(1,6,6,8,9,16)$ - second row in Table 5) does not belong to the set of feasible solutions, but meets the monotonicity condition, i.e. no confederation has received a smaller number of slots than confederations with fewer points. The $\operatorname{Core}(D P)$ allocation differs significantly from allocation $a$ but only for the smallest (OFC) and the largest (UEFA) 
Table 5 Core $(D P)$ for FIFA World Cup 2026

\begin{tabular}{lllllll}
\hline & OFC & CONCACAF & CONMEBOL & AFC & CAF & UEFA \\
\hline Value & 1211 & 9296 & 10,807 & 11,773 & 19,721 & 34,836 \\
$a$ & 1 & 6 & 6 & 8 & 9 & 16 \\
$\min$ & 1 & 6 & 6 & 6 & 7 & 8 \\
$\max$ & 7 & 8 & 8 & 8 & 11 & 16 \\
$\operatorname{Core}(D P)$ & 4 & 7 & 7 & 7 & 9 & 12 \\
\hline
\end{tabular}

confederation. In the case of CAF the number of slots in $\operatorname{Core}(D P)$ is equal to the number of slots in $a$, for the remaining confederations the difference is 1 .

\section{Conclusions}

In many practical applications constraints resulting from the conditions of degressively proportional allocation allow to numerically generate the set of all possible solutions. As a result, one can also define divisions which favor and disfavor, in a sense explained in this paper, ordered sets of agents. Hence, it is possible to indicate neutral divisions interpreted as most acceptable during negotiations leading to the selection of one division out of many possible allocations. The proposed iterative algorithm generates the set which can be considered as neutral as possible. The set can be a basis for efficient and reduced negotiations under transparent and equal conditions for all participants in division.

As shown in the example analyzed in the paper of how seats in the European Parliament of the first term were allocated, the actual allocation of goods assigned to individual countries can significantly diverge from the core of the set of all feasible solutions. What is more, the accepted solution in this example was the element of the eleventh core, i.e. 30 iterations before the end of algorithm. The situations of this type may evidence a stronger position of some agents in negotiations or their incomplete knowledge about available potential gains. The reduction of the cardinality of the set under analysis during negotiations makes this incompleteness smaller and streamlines the selection of a "compromise" solution. The same applies to the other examples. Furthermore, as the example of allocation of slots for FIFA World Cup shows, a unique solution can be found.

In perspective of further research, a major role can be played by those allocation sets for which a set with a single element is generated by the algorithm proposed in the paper. In this case, as suggested by the example presented in Table 2 in Sect. 5.1, the proposed algorithm can return the same outcome as the intuitive allocation, applied in practice for a long time, that is based on the Maimonides' rule. The method that can be applied to solve this problem comes from the well-known idea of transferring goods between agents. 
Author contributions Equal contributions of all authors.

Funding Not applicable.

Code availability Not applicable.

Availability of data and material Not applicable.

\section{Declarations}

Conflict of interest Not applicable.

Open Access This article is licensed under a Creative Commons Attribution 4.0 International License, which permits use, sharing, adaptation, distribution and reproduction in any medium or format, as long as you give appropriate credit to the original author(s) and the source, provide a link to the Creative Commons licence, and indicate if changes were made. The images or other third party material in this article are included in the article's Creative Commons licence, unless indicated otherwise in a credit line to the material. If material is not included in the article's Creative Commons licence and your intended use is not permitted by statutory regulation or exceeds the permitted use, you will need to obtain permission directly from the copyright holder. To view a copy of this licence, visit http://creativecommons.org/licen ses/by/4.0/.

\section{References}

Agenor, P.-R., \& Neanidis, K. (2011). The allocation of public expenditure and economic growth. The Manchester School, 79(4), 899-931

Arora, S., Gupta, D., \& Jain, S. (2016). An improvement in LaRSA and its implementation on allocation of seats to categories in an organization. Indian Journal of Computer Science and Engineering, 6, $182-189$

Barbera, S., \& Jackson, M. O. (2006). On the weights of nations: Assigning voting weights in a heterogeneous union. Journal of Political Economy, 114(2), 317-339

Beisbart, C., \& Bovens, L. (2007). Welfarist evaluations of decision rules for boards of representatives. Social Choice and Welfare, 29(4), 581-608

Cegiełka, K., Dniestrzański, P., Łyko, J., \& Maciuk, A. (2019a). Ordering and priority of entitlements in the problem of degressively proportional allocation. In Economic and social development. 41st international scientific conference on economic and social development, Book of Proceedings, Belgrade, Varazdin Development and Entrepreneurship Agency, 133-140

Cegiełka, K., Dniestrzański, P., Łyko, J., Maciuk, A., \& Rudek, R. (2017). On ordering a set of degressively proportional apportionments. Transactions on Computational Collective Intelligence, XXVII, 53-62

Cegiełka, K., Łyko, J., \& Rudek, R. (2019). Beyond the Cambridge Compromise algorithm towards degressively proportional allocations. Operational Research, 19, 317-332

Charvat, J. (2019). The politics of (mal)apportionment and member state representation in the European Parliament, Conference V4/EU, Summer Scholl 2019, Budapest.

Chen, Z., Hu, Z., \& Tang, Q. (2017). Allocation inequality in cost sharing problem. Journal of Mathematical Economics 91, 111-120. https://doi.org/10.2139/ssrn.2999238

Delgado-Márquez, B., Kaeding, M., \& Palomares, A. (2013). A more balanced composition of the European Parliament with degressive proportionality. European Union Politics, 14, 458-471

Dniestrzański, P. (2014). Proposal for measure of degressive proportionality. Procedia Social and Behavioral Sciences, 110, 140-147

Dniestrzański, P. (2014). The proposal of allocation of seats in the European Parliament: The shifted root. Procedia Social and Behavioral Sciences, 124, 536-543 
Dniestrzański, P., \& Łyko, J. (2014). Influence of boundary conditions of degressively proportional division on the potential application of proportional rules. Procedia Social and Behavioral Sciences, $109,722-729$

Duff, A. (2012). Finding the balance of power in a post-national democracy. Mathematical Social Sciences, 63(2), 74-77

Florek, J. (2012). A numerical method to determine a degressive proportional distribution of seats in the European Parliament. Mathematical Social Sciences, 63(2), 121-129

Grimmett, G. (2012). European apportionment via the Cambridge Compromise. Mathematical Social Sciences, 63(2), 68-73

Grimmett, G. R., Oelbermann, K. F., \& Pukelsheim, F. (2012). A power-weighted variant of the EU27 Cambridge Compromise. Mathematical Social Sciences, 63(2), 136-140

Habermas, J. (2017). Citizen and state equality in a supranational political community: degressive proportionality and the Pouvoir Constituant Mixte. Journal of Common Market Studies, 55, 171-182

Haman, J. (2007). Degresywnie proporcjonalny podział mandatów w Parlamencie Europejskim [Degressively proportional division of seats in the European Parliament]. Decyzje, 8, 53-78

Haman, J. (2017). The concept of degressive and progressive proportionality and its normative and descriptive applications. Studies of Logic, Grammar and Rhetoric, 50(1), 67-91

Koriyama, Y., Macé, A., Treibich, R., \& Laslier, J. F. (2013). Optimal apportionment. Journal of Political Economy, 121(3), 584-608

Kurz, S., Maaser, N., \& Napel, S. (2017). On the democratic weights of nations. Journal of Political Economy, 125(5), 1599-1634

Laslier, J. F. (2012). Why not proportional? Mathematical Social Sciences, 63(2), 90-93

Leeman, L., \& Mares, I. (2014). The adoption of proportional representation. The Journal of Politics, 76(2), 461-478

Łyko, J., \& Łyko, E. (2020). The composition of the European Parliament during the 2019-2024 term in light of legal provisions and the rules of fair distribution. In Eurasian economic perspectives. Proceedings of the 26th and 27th Eurasia business and economics society conferences, Eurasian Studies in Business and Economics, vol. 14, nr 1, Cham, Springer, 363-374

Łyko, J., \& Rudek, R. (2013). A fast exact algorithm for the allocation of sets for the EU Parliament. Expert Systems with Applications, 40, 5284-5291

Łyko, J., \& Rudek, R. (2017). Operations research methods in political decisions: A case study on the European Parliament composition. Computational and Mathematical Organization Theory, 23, 572-586

Macé, A., \& Treibich, R. (2012). Computing the optimal weights in a utilitarian model of apportionment. Mathematical Social Sciences, 63(2), 141-151

Martinez-Aroza, J., \& Ramirez-Gonzalez, V. (2008). Several methods for degressively proportional allotments. A case study. Mathematical and Computer Modelling, 48, 1439-1445

Miller, N. R. (2012). Why the Electoral College is good for political science (and public choice). Public Choice, 150(1-2), 1-25

Moberg, A. (2012). EP seats: The politics behind the math. Mathematical Social Sciences, 63(2), 78-84

Papathanasiou, J., \& Ploskas, N. (2018). Multiple criteria decision aid. Methods, examples and python implementations, Springer optimization and its applications, vol. 136. Springer.

Pham, N. A. (2019). Lorenz comparison between increasing serial and Shapley value cost-sharing rules. Economic Letters, 179, 49-52

Policy Department for Citizens' Rights and Constitutional Affairs. (2017). PE583.117 of 30 January 2017. The Composition of the European Parliament.

Pukelsheim, F. (2010). Putting citizens first: Representation and power in the European Union. In M. Cichocki \& K. Życzkowski (Eds.), Institutional design and voting power in the European Union. (pp. 235-253). Ashgate.

Pukelsheim, F. (2014). Proportional representation. Apportionment methods and their applications. Springer.

Pukelsheim, F., \& Grimmett, G. R. (2018). Degressive representation of member states in the European Parliament 2019-24. Representation, 54(2), 147-158

Ramirez-Gonzalez, V. (2012). Seat distribution in the European Parliament according to the Treaty of Lisbon. Mathematical Social Sciences, 63(2), 130-135

Ramírez González, V., Martínez-Aroza, J., \& Márquez García, M. (2012). Spline methods for degressive proportionality in the composition of European Parliament. Mathematical Social Sciences, 63(2), $114-120$ 
Ramírez-González, V., Palomares, B. A., \& Márquez Garcia, M. (2006). Degressively proportional methods for the allotment of the European Parliament seats amongst the UE member States. In B. Simeone \& F. Pukelsheim (Eds.), Mathematics and democracy. Recent advances in voiting systems and collective choice. (pp. 205-220). Springer.

Rudek, R., \& Heppner, I. (2020). Efficient algorithms for discrete resource allocation problems under degressively proportional constraints. Expert Systems With Applications. https://doi.org/10.1016/j. eswa.2020.113293

Serafini, P. (2012). Allocation of the EU Parliament seats via integer linear programming and revised quotas. Mathematical Social Sciences, 63(2), 107-113

Słomczyński, W., \& Życzkowski, K. (2012). Mathematical aspects of degressive proportionality. Mathematical Social Sciences, 63(2), 94-101

$\mathrm{Xu}, \mathrm{X}$. (2001). The SIR method: A superiority and inferiority ranking method for multiple criteria decision making. European Journal of Operational Research, 131(3), 587-602

Yildirim, B. F., \& Mercangoz, B. A. (2020). Evaluating the logistics performance of OECD countries by using fuzzy AHP and ARAS-G. Eurasian Economic Review, 2020, 27-45

Young, H. P. (1994). Equity: In theory and practice. Princeton University Press.

Zhang, M., \& Zou, H. (1998). Fiscal decentralization, public spending, and economic growth in China. Journal of Public Economics, 67(2), 221-240

Publisher's Note Springer Nature remains neutral with regard to jurisdictional claims in published maps and institutional affiliations.

\section{Authors and Affiliations}

\section{Katarzyna Cegiełka ${ }^{1}$ D . Piotr Dniestrzański ${ }^{1}$ (D Janusz Łyko $^{1}$ (D) .

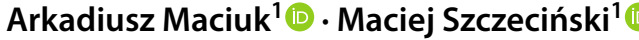

Piotr Dniestrzański

piotr.dniestrzanski@ue.wroc.pl

Janusz Łyko

janusz.lyko@ue.wroc.pl

Arkadiusz Maciuk

arkadiusz.maciuk@ue.wroc.pl

Maciej Szczeciński

maciej.szczecinski@ue.wroc.pl

1 Wroclaw University of Economics and Business, Komandorska st. 118/120, 53-345 Wrocław, Poland 